\title{
Plasma YKL-40 in the spectrum of neurodegenerative dementia
}

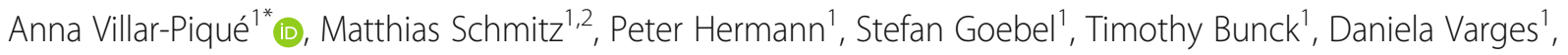
Isidre Ferrer ${ }^{3,4,5}$, Joachim Riggert ${ }^{6}$, Franc Llorens ${ }^{1,3,4^{*}+}$ and Inga Zerr ${ }^{1,2+}$

\begin{abstract}
Background: Increased plasma YKL-40 has been reported in Alzheimer's disease (AD), but its levels in other neurodegenerative diseases are unknown. Here, we aimed to investigate plasma YKL-40 in the spectrum of neurodegenerative dementias.
\end{abstract}

Methods: YKL-40 was quantified in the plasma of 315 cases, including healthy controls (HC), neurological disease controls (ND), AD, vascular dementia (VaD), frontotemporal dementia (FTD), sporadic Creutzfeldt-Jakob disease (CJD) and Lewy body dementia (LBD). Diagnostic accuracy in the differential diagnostic context and influence of age and gender was assessed.

Results: Highest YKL-40 levels were detected in CJD, followed by LBD, VaD, AD, FTD, ND and HC. YKL-40 was associated to age but not to sex. After controlling for age, YKL-40 was significantly elevated in CJD compared to HC $(p<0.001)$, ND, AD and $\mathrm{VaD}(p<0.01)$ and in LBD compared to HC $(p<0.05)$. In CJD, YKL-40 concentrations were significantly higher at late disease stages.

Conclusions: Plasma YKL-40 is significantly elevated in CJD regardless of clinical and genetic parameters, with moderate diagnostic accuracy in the discrimination from control cases. Our study discards a potential use of this biomarker in the differential diagnostic context but opens the possibility to be explored as a marker for CJD monitoring.

Keywords: YKL-40, CHI3L1, Neurodegenerative dementia, Biomarker, Prion diseases, Plasma

\section{Introduction}

YKL-40, also known as chitinase-3-like protein 1 (CHI3L1), is a secreted glycoprotein expressed in several tissues and involved in activation of the innate immune system and in cell processes in relation to extracellular matrix remodeling [1-3]. Cerebrospinal fluid (CSF) concentrations of YKL-40 are significantly increased in sporadic Creutzfeldt-Jakob disease (CJD) and Alzheimer's disease (AD), while other neurodegenerative dementias such as frontotemporal dementia (FTD), Lewy body dementia (LBD) and vascular dementia (VaD) show normal to slightly altered levels [4-6].

* Correspondence: avillar@gwdg.de; franc.llorens@gmail.com

${ }^{\dagger}$ Franc Llorens and Inga Zerr are equal senior authors.

${ }^{1}$ Department of Neurology, Clinical Dementia Center and National Reference Center for CJD Surveillance, University Medical School, Robert Koch 40, 37075 Göttingen, Germany

Full list of author information is available at the end of the article
However, the potential role of YKL-40 as blood-based biomarker in the differential diagnostic has not been explored. In $\mathrm{AD}$, plasma YKL-40 concentrations were reported to be increased, but with limited utility as a diagnostic marker [7] presenting moderate effect sizes according to meta-analysis studies [8]. The levels of plasma YKL-40 in other neurodegenerative dementias are unknown.

Here we evaluated the accuracy of plasma YKL-40 in the discrimination of neurodegenerative dementias from different etiologies.

\section{Methods \\ Samples \\ Blood was collected in plasma-EDTA tubes at the Department of Transfusion Medicine (healthy controls $(\mathrm{HC})$ ) and at the Department of Neurology-Clinical Dementia Center (neurological disease controls (ND),}


sporadic Creutzfeldt-Jakob disease (CJD), Alzheimer's disease (AD), frontotemporal dementia (FTD), Lewy body dementia (LBD) and vascular dementia $(\mathrm{VaD})$ ) in the Universitätsmedizin Göttingen (Germany) under same pre-analytical conditions.

The HC group was composed of healthy blood donors with absence of any relevant clinical finding. The ND group was composed of cases with neurological conditions not associated with neurodegenerative pathology diagnosed according to acknowledged standard neurologic clinical and para-clinical findings based on the International Classification of Diseases 10th Edition definitions. AD was diagnosed according to the National Institute on Aging-Alzheimer's Association workgroups (NIA-AA) criteria [9]. CJD cases were classified as probable or definite according to diagnostic consensus criteria $[10,11]$. The Lewy body dementia (LBD) group included dementia with Lewy bodies (DLB) and Parkinson's disease dementia (PDD) cases. The diagnosis of DLB was based on the criteria of McKeith [12]. PDD diagnosis was based on the task force of the Movement Disorder Society (MDS) criteria [13]. FTLD was diagnosed according to the International Behavioural Variant FTD Criteria Consortium for bvFTD [14]. VaD diagnosis was based on clinical and radiological criteria as described by Roman (National Institute of Neurological and Communicative Disorders and Stroke and the Alzheimer's Disease and Related Disorders Association (NINDS-AIREN)) [15]. In CJD cases, genetic testing of the codon 129 polymorphism of prion protein gene (PRNP) was conducted as described before [16]. For disease stage, samples were stratified in three categories according to whether they underwent blood uptake in the first (early) (time of blood uptake to disease onset/ total duration of the disease $<0.33$ ), second (middle) $(0.33-0.66)$, or third (last) $(>0.66)$ stage of the disease. Disease duration was recorded as the time (in months) from symptom onset to the death of the patient.

\section{Plasma YKL-40 quantification}

Plasma YKL-40 was measured with the MicroVue YKL40 EIA ELISA kit from Quidel following the manufacturer's instructions. Samples were diluted 1:2 to 1:4. Intra- and inter-assay coefficient of variation was $9 \%$ and $14 \%$, respectively.

\section{Statistical analysis}

We performed one-way analysis of variance followed by Tukey correction to compare age between disease groups. Association between YKL-40 levels and age was explored with Pearson correlation coefficient. We logtransformed YKL-40 concentration to obtain a normal distribution and applied analysis of covariance followed by Tukey correction to assess the differences between groups controlling for age as covariant. Association between YKL-40 and sex was investigated with the MannWhitney test. Receiver operating characteristic (ROC) curve and areas under the curve (AUC) with 95\% confidence intervals $(95 \% \mathrm{CI})$ were calculated to assess diagnostic accuracies between diagnostic groups. All analyses were performed using GraphPad Prism software and multcomp package in $\mathrm{R}$ [17].

\section{Results}

The study population consisted of 315 plasma agematched cases with the exception of $\mathrm{VaD}$ cases that were significantly older than HC $(p<0.01)$ (Table 1$)$. In the total population, YKL-40 was associated with age $(\mathrm{rho}=0.21$, $p<0.001)$ (Fig. 1a), but not with sex $(p=0.20)$ (Fig. 1b). Mean YKL-40 concentrations were higher in neurodegenerative dementias compared to $\mathrm{HC}(84 \mathrm{ng} / \mathrm{mL})$ and $\mathrm{ND}$ $(95 \mathrm{ng} / \mathrm{mL})$, with highest concentrations detected in CJD (189 $\mathrm{ng} / \mathrm{mL})$, followed by LBD (167 ng/mL), VaD (140 ng/ $\mathrm{mL}), \mathrm{AD}(133 \mathrm{ng} / \mathrm{mL})$ and FTD $(125 \mathrm{ng} / \mathrm{mL})$ (Table 1). After age correction, YKL-40 levels appeared significantly higher in CJD versus $\mathrm{HC}(p<0.001), \mathrm{ND}, \mathrm{AD}$ and $\mathrm{VaD}$ $(p<0.01)$ and in LBD compared to HC $(p<0.05)$ (Fig. 1c).

Table 1 Demographic, plasma YKL-40 concentrations and diagnostic accuracy in discrimination from healthy controls and neurological disease groups

\begin{tabular}{|c|c|c|c|c|c|c|c|}
\hline & \multirow[t]{2}{*}{$n$} & \multirow{2}{*}{$\begin{array}{l}\text { Sex } \\
(f / m)\end{array}$} & \multirow{2}{*}{$\begin{array}{l}\text { Age } \\
\text { (years) }\end{array}$} & \multicolumn{2}{|c|}{$\mathrm{YKL}-40(\mathrm{ng} / \mathrm{mL})$} & \multicolumn{2}{|l|}{ AUC (95\% Cl) } \\
\hline & & & & $\overline{M e a n}+\mathrm{SD}$ & $95 \% \mathrm{Cl}$ & vs. HC & vs. ND \\
\hline $\mathrm{HC}$ & 70 & $22 / 48$ & $66 \pm 5$ & $84 \pm 84$ & 63-104 & & $0.64(0.53-0.74)$ \\
\hline ND & 44 & $26 / 18$ & $66 \pm 12$ & $95 \pm 61$ & $76-114$ & $0.64(0.53-0.74)$ & \\
\hline$A D$ & 50 & $25 / 25$ & $69 \pm 10$ & $133 \pm 110$ & $102-164$ & $0.62(0.51-0.73)$ & $0.55(0.43-0.67)$ \\
\hline $\mathrm{VaD}$ & 22 & $8 / 14$ & $72 \pm 10^{* *}$ & $140 \pm 150$ & $73-206$ & $0.56(0.40-0.72)$ & $0.51(0.34-0.68)$ \\
\hline FTD & 17 & $11 / 6$ & $68 \pm 12$ & $125 \pm 108$ & 69-181 & $0.65(0.50-0.80)$ & $0.55(0.38-0.73)$ \\
\hline CJD & 78 & $51 / 27$ & $67 \pm 8$ & $189 \pm 167$ & $151-227$ & $0.81(0.74-0.88)$ & $0.72(0.63-0.81)$ \\
\hline LBD & 34 & $13 / 21$ & $69 \pm 8$ & $167 \pm 157$ & $112-222$ & $0.70(0.59-0.81)$ & $0.63(0.49-0.76)$ \\
\hline
\end{tabular}

Number of cases $(n)$, sex (female $[\mathrm{f}] /$ male $[\mathrm{m}]$ ), age in years (mean values \pm standard deviation (SD)), YKL-40 plasma concentrations (mean values \pm SD) and $95 \%$ confidence interval $(95 \% \mathrm{CI})$ ) and area under the curve (AUC) with $95 \% \mathrm{Cl}$ values for each dementia diagnostic comparison versus $\mathrm{HC}$ and $\mathrm{ND}$ are indicated ${ }^{* *} p<0.01$ 


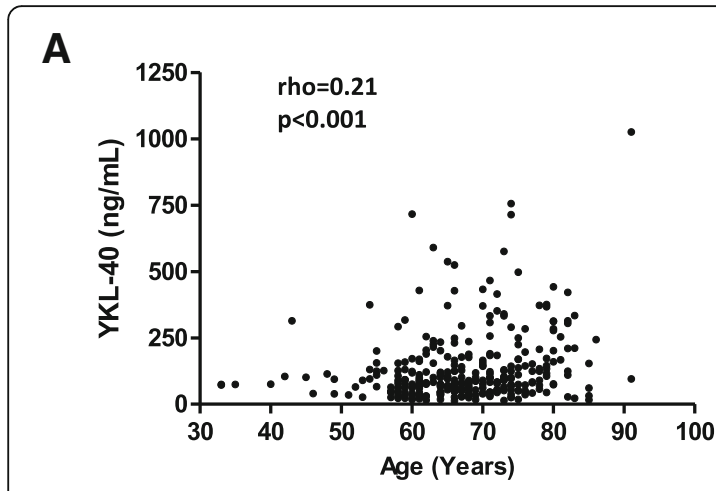

B
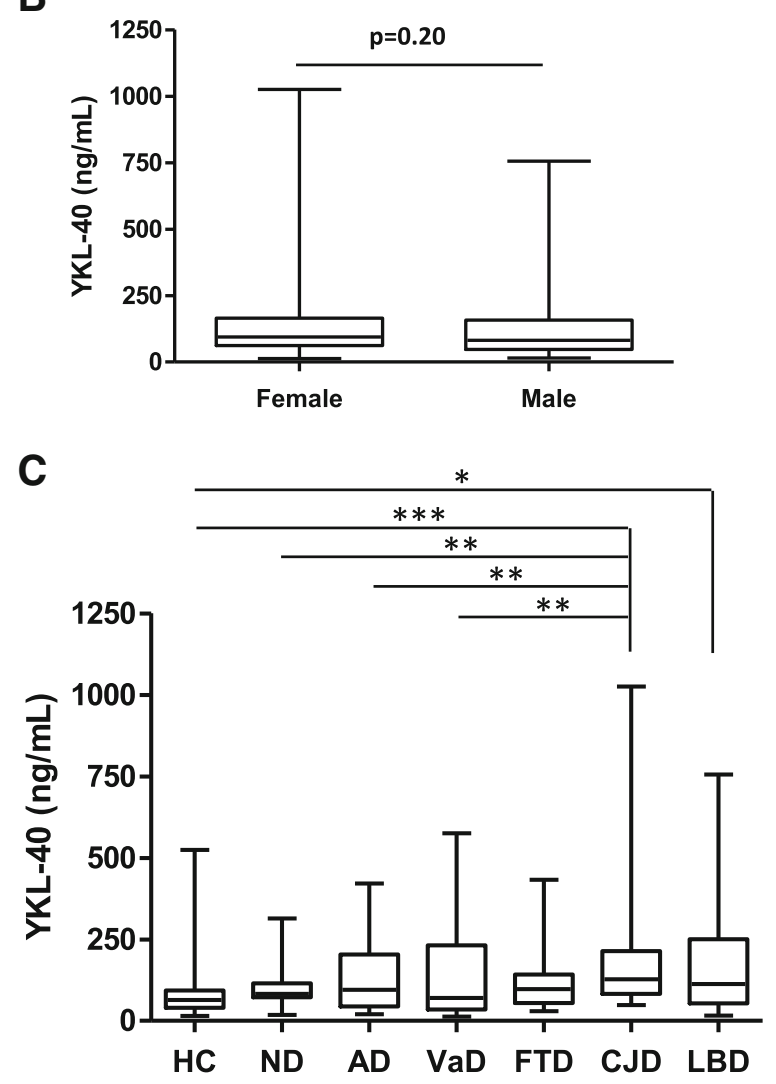

Fig. 1 Plasma YKL-40 in the differential diagnostic context of neurodegenerative dementia and association with demographics. a Association between YKL-40 concentration and age at disease onset in the study population. $\mathbf{b} \mathrm{YKL}-40$ concentration stratified by sex. $\mathbf{c}$ YKL-40 concentration in HC, ND, AD, VaD, FTD, CJD and LBD. After age correction, $\mathrm{YKL}-40$ was significantly elevated in CJD compared to $\mathrm{HC}(p<0.001), \mathrm{ND}, \mathrm{AD}$ and $\mathrm{VaD}(p<0.01)$ and in LBD compared to $\mathrm{HC}(p<0.05) .{ }^{*} p<0.05,{ }^{* *} p<0.01$ and ${ }^{* * *} p<0.001$

Area under the curve (AUC) for the discrimination of neurodegenerative dementia groups from controls ranged from 0.56 to 0.81 for $\mathrm{HC}$ and from 0.51 to 0.72 for ND, indicating low diagnostic value except in the discrimination between CJD from $\mathrm{HC}$, which achieved a moderate value (0.81) (Table 1).
Since the CJD group presented the most significant YKL-40 increase compared to other diagnostic groups, we conducted further investigations. We stratified CJD cases depending on the codon 129 polymorphism of PRNP gene, but no differences in YKL-40 concentrations were detected between methionine/methionine (MM) (165 ng/mL, $n=49)$, methionine/valine (MV) (212 ng/ $\mathrm{mL}, n=10)$ and valine/valine (VV) $(173 \mathrm{ng} / \mathrm{mL}, n=13)$ cases (Fig. 2a). Similarly, no differences were detected between the most prevalent CJD subtypes, MM1 (136 $\mathrm{ng} / \mathrm{mL}, n=34)$ and VV2 $(156 \mathrm{ng} / \mathrm{mL}, n=12)(p=0.32)$ (Fig. 2b). To assess the potential alteration of plasma YKL-40 along disease progression, CJD cases were stratified in three groups according to the stage of the disease where the blood was collected (1/early: $n=13,2 /$ middle: $n=16$ and 3/late: $n=40$ disease stage). We found a significant increase of YKL between late disease stage (215 $\mathrm{ng} / \mathrm{mL})$ and early disease stage $(104 \mathrm{ng} / \mathrm{mL}, p=0.036)$ (Fig. 2c). However, lack of association between YKL-40 concentrations and disease duration was present in the CJD group ( $p=0.32$ ) (Fig. $2 \mathrm{~d})$.

\section{Discussion}

Many efforts are devoted to discover blood-based clinical biomarkers for the diagnosis of neurodegenerative diseases. In this search, plasma YKL-40 has been positioned as a promising candidate and a significant elevation was reported in mild AD-type dementia and early AD compared to controls $[7,18]$, but the significance of these findings in the differential diagnostic context of neurodegenerative dementias was unknown. Although we were able to detect increased mean plasma YKL-40 concentrations in $\mathrm{AD}$ compared to controls, the comparative analysis in different dementia conditions indicated that only statistically significant alterations were observed between LBD and HC and between CJD and $\mathrm{HC}, \mathrm{ND}, \mathrm{AD}$ and $\mathrm{VaD}$. Resultant AUC values were low $(<0.8)$, except from that obtained between CJD and $\mathrm{HC}$, which reached 0.81. However, this value was still far from that rendered by CSF YKL-40 between CJD and neurological controls (AUC $=0.92)$ [4].

Plasma YKL-40 levels were influenced by age but not by sex, as it is the case of CSF YKL-40 [7]. Within the CJD group, no differences in YKL-40 levels were detected based on the most prevalent clinical subtypes, MM1 and VV2, even though they display different clinic-pathological outcomes. Similarly, no association between plasma YKL-40 and codon 129 polymorphism of PRNP either disease duration was observed, in agreement with the data obtained for CSF YKL-40 [4]. Thus, the moderate AUC value achieved in the discrimination of CJD from $\mathrm{HC}$ presents robustness in front of clinical heterogeneity. By contrast, we found that CJD cases at early disease stages present significantly lower values than those at late stage. Therefore, although the value 


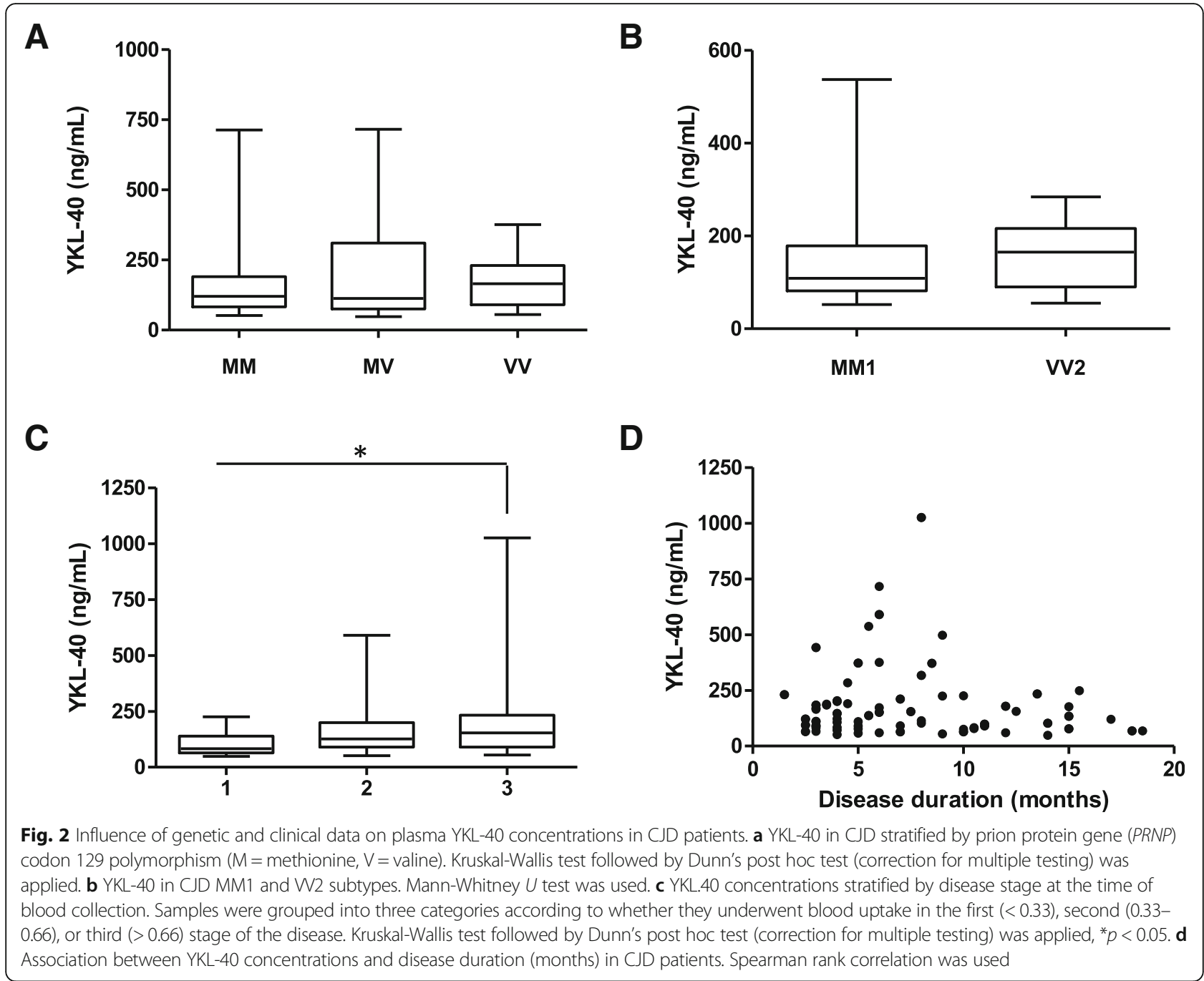

of plasma YKL-40 as diagnostic biomarker is rather limited and our data do not support its usefulness in the differential diagnostic context, this marker may serve in the evaluation of disease progression and monitoring of potential therapeutic interventions.

Being CSF YKL-40 considered a sound marker of neuro-inflammation, its rise in CJD, $\mathrm{AD}$ and other neurodegenerative diseases characterized by neuroinflammatory profile is expected $[4-6,19]$. However, the weak correlation between CSF and plasma YKL-40 previously reported $[7,20]$ and the fact that YKL-40 levels in blood are not altered in diagnostic groups where CSF levels are so [20] indicates that alternative mechanisms, other than direct CSF-blood exchange, might exist to explain the regulation of plasma YKL-40 herein observed. In the brain, the expression of YKL-40 upon inflammation conditions is mainly attributed to reactive astrocytes [7, 21-23]. In the case of CJD, the presence of perivascular astrocytes was detected in cortical regions [4]. Thus, it is tempting to speculate that, upon damage of the brain blood vessels or impairment of the blood-brain barrier, which is common in many neurodegenerative diseases [24], this subset of astrocytes could release brain-derived YKL-40 in the blood. In this case, the increase of YKL-40 in plasma at advanced CJD stage that we observed may reflect, not only the degree of neuro-inflammation, but also suspected damage in brain blood vessels. Further investigations will be necessary to demonstrate this hypothesis and clarify the origin of YKL-40 in the plasma of patients with neurodegenerative dementia.

\section{Conclusions}

Altogether, our study indicates that plasma YKL-40 may contribute to the diagnosis of CJD regardless of clinical heterogeneity, but it should be combined with other blood-based biomarkers to increase its diagnostic performance. Contrarily, our data do not support the use of this marker in the challenging differential diagnostic context of neurodegenerative dementias. Additionally, a potential use as a CJD progression marker is envisaged. 


\section{Abbreviations}

AD: Alzheimer's disease; AUC: Area under the curve; CJD: Creutzfeldt-Jakob disease; CSF: Cerebrospinal fluid; FTD: Frontotemporal dementia; HC: Healthy controls; LBD: Lewy body dementia; ND: Neurological disease controls; VaD: Vascular dementia

\section{Acknowledgements}

Not applicable.

\section{Authors' contributions}

AV-P, FL and IZ participated in the conception and design of the study. AV-P, MS, PH, SG, TB, DV, IF, JR, FL and IZ participated in the acquisition and analysis of data. AV-P and FL drafted the manuscript and the figures. All authors read and approved the final manuscript.

\section{Funding}

This study was funded by the Spanish Ministry of Health - Instituto Carlos III (Miguel Servet programme - CP/00041) to FL and by the Robert Koch Institute through funds from the Federal Ministry of Health (grant No, 1369341 ) to IZ. This project was also funded at $65 \%$ by the Fondo Europeo de Desarrollo Regional (FEDER) through the Interreg V-A España-Francia-Andorra (POCTEFA 2014-2020) programme.

\section{Availability of data and materials}

The datasets used during the current study are available from the corresponding authors on reasonable request.

\section{Ethics approval and consent to participate}

Written informed consent was obtained from all study participants or their legal guardians. The study was conducted according to the revised Declaration of Helsinki and Good Clinical Practice guidelines and was approved by all local Ethics committees (Reference numbers 11/11/93, 5/09/ 08, 9/06/08, 19/11/09, Universitätsmedizin Göttingen, Germany).

\section{Consent for publication}

$$
\text { Not applicable. }
$$

\section{Competing interests}

The authors declare that they have no competing interests.

\section{Author details}

'Department of Neurology, Clinical Dementia Center and National Reference Center for CJD Surveillance, University Medical School, Robert Koch 40, 37075 Göttingen, Germany. ${ }^{2}$ German Center for Neurodegenerative Diseases (DZNE), Göttingen, Germany. ${ }^{3}$ Network Center for Biomedical Research in Neurodegenerative Diseases, (CIBERNED), Institute Carlos III, Ministry of Health, Feixa Llarga s/n, L'Hospitalet de Llobregat, 08907 Barcelona, Spain. ${ }^{4}$ Bellvitge Biomedical Research Institute (IDIBELL), Hospitalet de Llobregat, Spain. ${ }^{5}$ Department of Pathology and Experimental Therapeutics, University of Barcelona, Hospitalet de Llobregat, Spain. ${ }^{6}$ Department of Transfusion Medicine, University Medical School, Göttingen, Germany.

Received: 4 December 2018 Accepted: 25 June 2019

Published online: 12 July 2019

\section{References}

1. Rathcke CN, Vestergaard H. YKL-40, a new inflammatory marker with relation to insulin resistance and with a role in endothelial dysfunction and atherosclerosis. Inflamm Res. 2006;55(6):221-7.

2. Shao R, Hamel K, Petersen L, Cao QJ, Arenas RB, Bigelow C, et al. YKL-40, a secreted glycoprotein, promotes tumor angiogenesis. Oncogene. 2009;28: 4456-68 Available from: http://www.pubmedcentral.nih.gov/articlerender. fcgi?artid=2795793\&tool=pmcentrez\&rendertype=abstract.

3. Johansen JS. Studies on serum YKL-40 as a biomarker in diseases with inflammation, tissue remodelling, fibroses and cancer. Dan Med Bull. 2006;53: 172-209.

4. Llorens F, Thüne K, Tahir W, Kanata E, Diaz-Lucena D, Xanthopoulos K, et al. YKL-40 in the brain and cerebrospinal fluid of neurodegenerative dementias. Mol Neurodegener. 2017;12(1):83.

5. Wennström M, Surova Y, Hall S, Nilsson C, Minthon L, Hansson O, et al. The inflammatory marker YKL-40 is elevated in cerebrospinal fluid from patients with Alzheimer's but not Parkinson's disease or dementia with Lewy bodies. PLoS One. 2015:10(8):e0135458.

6. Janelidze S, Hertze J, Zetterberg H, Landqvist Waldo M, Santillo A, Blennow $\mathrm{K}$, et al. Cerebrospinal fluid neurogranin and YKL-40 as biomarkers of Alzheimer's disease. Ann Clin Transl Neurol. 2015;3:n/a Available from: http://doi.wiley.com/10.1002/acn3.266.

7. Craig-Schapiro R, Perrin RJ, Roe CM, Xiong C, Carter D, Cairns NJ, et al. YKL40: a novel prognostic fluid biomarker for preclinical Alzheimer's disease. Biol Psychiatry. 2010;68:903-12

8. Olsson B, Lautner R, Andreasson U, Öhrfelt A, Portelius E, Bjerke M, et al. CSF and blood biomarkers for the diagnosis of Alzheimer's disease: a systematic review and meta-analysis. Lancet Neurol. 2016;15(7):673-84.

9. McKhann GM, Knopman DS, Chertkow H, Hyman BT, Jack CR, Kawas CH, et al. The diagnosis of dementia due to Alzheimer's disease: recommendations from the National Institute on Aging-Alzheimer's Association workgroups on diagnostic guidelines for Alzheimer's disease. Alzheimers Dement. 2011; 7:263-9.

10. Zerr I, Kallenberg K, Summers DM, Romero C, Taratuto A, Heinemann U, et al. Updated clinical diagnostic criteria for sporadic Creutzfeldt-Jakob disease. Brain. 2009:132:2659-68.

11. Parchi P, De Boni L, Saverioni D, Cohen ML, Ferrer I, Gambetti P, et al. Consensus classification of human prion disease histotypes allows reliable identification of molecular subtypes: an inter-rater study among surveillance centres in Europe and USA. Acta Neuropathol. 2012;124:517-29.

12. McKeith IG, Boeve BF, Dickson DW, Halliday G, Taylor J-P, Weintraub D, et al. Diagnosis and management of dementia with Lewy bodies: fourth consensus report of the DLB consortium. Neurol Int. 2017;89:88-100.

13. Emre M, Aarsland D, Brown R, Burn DJ, Duyckaerts C, Mizuno Y, et al. Clinical diagnostic criteria for dementia associated with Parkinson's disease. Mov Disord. 2007;22(12):1689-707.

14. Rascovsky K, Hodges JR, Knopman D, Mendez MF, Kramer JH, Neuhaus J, et al. Sensitivity of revised diagnostic criteria for the behavioural variant of frontotemporal dementia. Brain. 2011;134:2456-77.

15. Román GC, Tatemichi TK, Erkinjuntti T, Cummings JL, Masdeu JC, Garcia JH, et al. Vascular dementia: diagnostic criteria for research studies. Report of the NINDS-AIREN International Workshop. Neurology. 1993;43:250-60.

16. Windl O, Giese A, Schulz-Schaeffer W, Zerr I, Skworc K, Arendt S, et al. Molecular genetics of human prion diseases in Germany. Hum Genet. 1999;105:244-52.

17. Hothorn T, Bretz F, Westfall P. Simultaneous inference in general parametric models. Biom J. 2008;50(3):346-63.

18. Choi J, Lee H-W, Suk K. Plasma level of chitinase 3-like 1 protein increases in patients with early Alzheimer's disease. J Neurol. 2011;258:2181-5 Available from: http://link.springer.com/10.1007/s00415-011-6087-9.

19. Alcolea D, Carmona-Iragui M, Suarez-Calvet M, Sanchez-Saudinos MB, Sala I, Anton-Aguirre S, et al. Relationship between beta-secretase, inflammation and core cerebrospinal fluid biomarkers for Alzheimer's disease. J Alzheimers Dis. 2014;42:157-67 Available from: http://content.iospress.com/ articles/journal-of-alzheimers-disease/jad140240.

20. Oeckl P, Weydt P, Steinacker P, Anderl-straub S, Nordin F, Volk AE, et al. Different neuroinflammatory profile in amyotrophic lateral sclerosis and frontotemporal dementia is linked to the clinical phase. J Neurol Neurosurg Psychiatry. 2018;90(1): 4-10.

21. Bonneh-Barkay D, Wang G, Starkey A, Hamilton RL, Wiley CA. In vivo CHI3L1 (YKL-40) expression in astrocytes in acute and chronic neurological diseases. J Neuroinflammation. 2010;7:34 Available from: http://www.pubmedcentral.nih. gov/articlerender.fcgi?artid=2892443\&tool=pmcentrez\&rendertype=abstract.

22. Bonneh-Barkay D, Bissel SJ, Kofler J, Starkey A, Wang G, Wiley CA. Astrocyte and macrophage regulation of $Y K L-40$ expression and cellular response in neuroinflammation. Brain Pathol. 2012:22:530-46.

23. Querol-Vilaseca M, Colom-Cadena M, Pegueroles J, San Martín-Paniello C, Clarimon J, Belbin O, et al. YKL-40 (chitinase 3-like I) is expressed in a subset of astrocytes in Alzheimer's disease and other tauopathies. J Neuroinflammation. 2017;14(1):118.

24. Sweeney MD, Sagare AP, Zlokovic BV. Blood-brain barrier breakdown in Alzheimer disease and other neurodegenerative disorders. Nat Rev Neurol. 2018:14(3):133-50

\section{Publisher's Note}

Springer Nature remains neutral with regard to jurisdictional claims in published maps and institutional affiliations. 